\title{
Combined Anatomic Reconstruction of the Anterior Cruciate and Anterolateral Ligaments, How I do it
}

\author{
N. Maffullii ${ }^{1,2,3}$, P. Colombet ${ }^{4}$, F. Oliva ${ }^{1}$, A. Oliviero ${ }^{1}$ \\ 1 Department of Musculoskeletal Disorders, Faculty of Medicine and Surgery, University of Salerno, Salerno, Italy \\ 2 Centre for Sports and Exercise Medicine, Barts and The London School of Medicine and Dentistry, Mile End \\ Hospital, 275 Bancroft Road, London E1 4DG, England \\ 3 Institute of Science and Technology in Medicine, Keele University School of Medicine, Thorburrow Drive, Stoke \\ on Trent ST4 7QB, England \\ ${ }^{4}$ Centre de Chirurgie Orthopédique et Sportive, 9 rue Jean Moulin, 33700 Bordeaux-Mérignac, France.
}

\author{
CORRESPONDING AUTHOR: \\ Nicola Maffulli \\ Department of Musculoskeletal Disorders, \\ Faculty of Medicine and Surgery, \\ University of Salerno, \\ Salerno, Italy \\ E-mail: n.maffulli@qmul.ac.uk \\ Telephone: + 442085677553 \\ Pbx: + 442082238930 \\ DOI: \\ 10.32098/mltj.03.2019.21
}

LEVEL OF EVIDENCE: 5

\begin{abstract}
SUMMARY
The sensation of residual rotational instability is found in more than $25 \%$ of patients undergoing anterior cruciate ligament (ACL) reconstruction. A potential cause of it could be injury of the antero-lateral ligament (ALL) of the knee. When the reconstruction of the ACL is associated with the reconstruction of the ALL, the risk of reoperation for graft failure, meniscal damage and knee osteoarthritis are significantly reduced. Furthermore, complications are similar to those encountered when ACL reconstruction is performed alone. We report the procedure developed by the senior authors (NM and PC) to reconstruct the ACL and the ALL in a minimally invasive anatomical fashion.
\end{abstract}

\section{KEY WORDS}

anterolateral soft tissue structures of the knee; extra-articular reconstruction; rotational instability.

\section{BACKGROUND}

Modern reconstruction techniques of the anterior cruciate ligament (ACL) produce reliably satisfactory results. However, at clinical examination, a degree of residual rotational stability is often found through a positive pivot shift test (1). Indeed, the sensation of residual rotational instability is found in more than $25 \%$ of patients undergoing ACL reconstruction (1).

The residual postoperative laxity of the knee could be caused by untreated anterolateral soft tissue injuries (2). Extra-articular repair or reconstruction of the lateral knee anatomy should therefore be considered.

Hughston et al (3), as early as 1976, had shown that acute and chronic rotational instability are associated with lesions of the middle third of the lateral knee joint capsule, and may be combined with ACL lesion.

In the past decade, combined intra- and extra-articular reconstruction procedures have been proposed to reduce anterolateral rotational instability (ALRI). Most extra-articular procedures collectively fall within the category of lateral extra-articular tenodesis (LET) procedures (4).

The extra-articular procedures acting mechanically on the periphery of the joint prevent the subluxation of the tibial plateau and protect the graft of the ACL (5). However, the popularity of LET procedures has diminished over time as they are non-anatomical, leading to early osteoarthritis from excessive overcostraint (6).

The combined ACL and ALL reconstruction has improved the rate of return to sport and reduced the risk of ACL graft ruptures $(7,8)$.

In 2007, Vieira (9) described the "capsulo-osseous" layer of the iliotibial band (ITB), naming it "the anterolateral ligament". Claes et al. (10), following a thorough study on 41 cadavers, described the ALL as an independent anatomical structure, reporting the sites of origin and insertion. There is a close correlation between the ALL and the later- 
al collateral ligament (LCL). Indeed, between these there are connecting fibers. In addition, the femoral origin of the ALL is located a little anterior to the origin of the LCL (10). The ALL runs obliquely and distally, presenting along its course expansions of attachment to the lateral meniscus, reaches the anterolateral margin of the proximal epiphysis of the tibia, and inserts approximately in the middle between the tubercle of Gerdy and the head of the fibula, separately from the iliotibial band (ITB).

Since 2013, great attention has been paid to the ALL; numerous studies have been performed to better understand its anatomy, biomechanics and clinical relevance. Monaco et al. (11), in a cadaver experiment, cutting the ALL, showed increasing tibial rotation which could be related to the pivot shift phenomenon. About 3 years later, a further by study Parsons et al. (12) showed that ALL acts as a stabilizer of internal rotation when the knee is flexed to more than $35^{\circ}$ We report the procedure developed by the senior authors to reconstruct the ACL and the ALL in a minimally invasive anatomical fashion.

\section{INDICATION}

1. Injury of the ACL and ALL diagnosed clinically with a grade III pivot shift and confirmed by magnetic resonance imaging (MRI);

2. Segond fracture;

3. ACL revision surgery.

Patients involved in high energy pivot-contact sports activities

\section{SURGICAL PROCEDURE}

We performed our investigation ethically according to international standards and as required by the journal (13).

The patient is placed supine with the knee flexed at $90^{\circ}$, the knee is kept stable by a lateral support to the thigh and a sandbag under the foot. A marking pen is used to mark the fibular head, Gerdy's tubercle and the lateral femoral epicondyle, so as to easily identify the origin and insertion sites of the ALL (14). The femoral origin is placed about 5 $\mathrm{mm}$ posteriorly and proximally to the lateral collateral ligament (LCL), the tibial insertion is approximately between the head of the fibula and the tubercle of Gerdy, $5 \mathrm{~mm}$ posterior to the centre of Gerdy's tubercle, and $10 \mathrm{~mm}$ below the joint line. The attachment and insertion of the ALL covers an area of $65 \mathrm{~mm}^{2}$ each, and its length averages $40 \mathrm{~mm}$.

The anteromedial and anterolateral standard arthroscopic portals are established and a gravity-based saline solution is used for fluid distension of the joint. After routine arthroscopic examination, the gracilis and semitendinosus tendons are harvested through a vertical $2 \mathrm{~cm}$ long incision centred $2 \mathrm{~cm}$ distal and $2.5 \mathrm{~cm}$ medial to the medial margin of the anterior tibial tuberosity (ATT). Before graft collection with an open tendon stripper, tendon expansions are carefully dissected to prevent premature truncation. The semitendinosus tendon is tripled and the gracilis tendon is left long. The proximal portion of the graft, which includes the triplicate semitendinosus and the first part of the gracilis, is sutured using $n^{\circ} 1$ Ethibond sutures (Ethicon, Edinburgh, United Kingdom). The proximal $25 \mathrm{~mm}$ of the gracilis tendon graft is whipstitched using $n^{\circ} 1$ Ethibond sutures suture. (Figure 1)

A standard guide for PCL is introduced through the anterolateral portal and the femoral tunnel in an outside-in fashion is produced, so that the end of the tunnel is $5 \mathrm{~mm}$ posterior and proximal to the LCL, corresponding to the femoral origin of the ALL

The femoral tunnel enters the knee joint in the posterior third of Blumensaat's line, in the middle of the area of femoral origin of the ACL and exits $5 \mathrm{~mm}$ posterior and proximal to the LCL, in correspondence to the femoral origin of the ALL. The tibial tunnel is produced in an outside-inside fashion with a $55^{\circ}$ angled tibial guide, entering the knee joint in the anterior half of the middle third of the tibial plateau. On the AP view, the lateral margin of the tibial tunnel is at the centre of the intercondylar notch, which is the highest point of the intercondylar notch

A suture loop is used by passing a $n^{\circ} 2$ Ethibond suture (Ethicon, Edinburgh, United Kingdom) in a retrograde fashion from the femoral tunnel to the tibial tunnel, and used for graft passage.

The graft is passed, without detaching its tibial insertion, thereby improving its fixation and vascularization, through both tunnels so that the part of the graft constituted by the tripled semitendinosus and the distal portion of the gracilis reconstruct the ACL, while the remaining part of the gracilis tendon is used to reconstruct percutaneously the ALL.

\section{(Figure 2)}

The graft is secured into the tibial tunnel under tension as it exits the femoral tunnel, using a non-metallic round-head, cannulated $8 \times 25 \mathrm{~mm}$ interference screw (RCI, Smith \& Nephew Endoscopy, Andover, Massachusetts). Then, with the knee at $30^{\circ}$ of flexion the graft is tensioned again, and subsequently the femoral side is secured using a non-metallic round-head, cannulated $7 \times 25 \mathrm{~mm}$ interference screw (RCI, Smith \& Nephew Endoscopy, Andover, Massachusetts) in an outside-in direction (15).

The remaining part of the graft, consisting of the free end of the gracilis tendon emerging from the femoral tunnel, is used to reconstruct the ALL.

For the ALL reconstruction, a tibial tunnel $(30 \mathrm{~mm}$ long) is produced according to the following steps. 

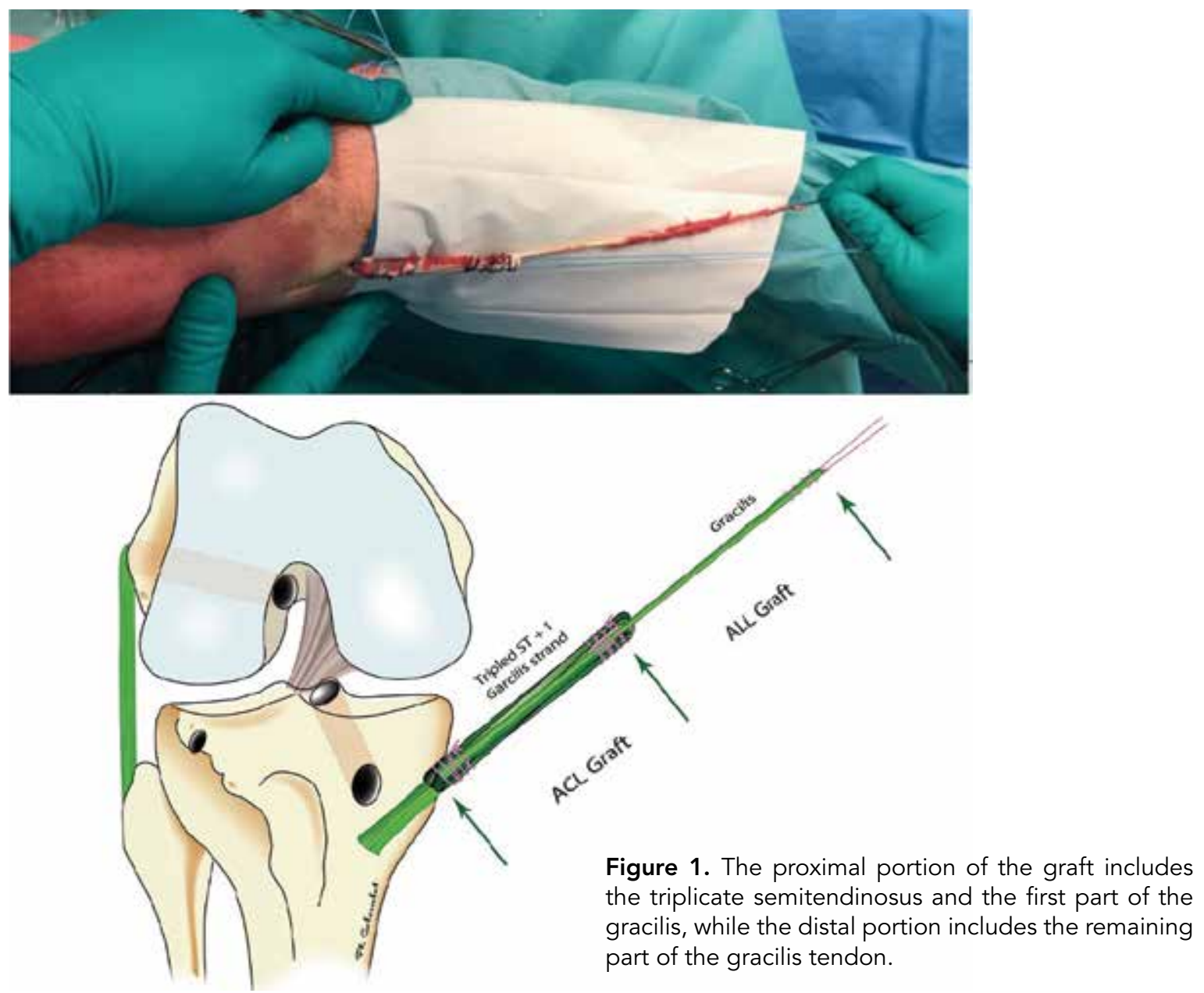

A small incision is performed on the tibial insertion of the ALL about $20 \mathrm{~mm}$ posterior to the centre of the Gerdy's tubercle, and $10 \mathrm{~mm}$ below the joint line. The guidewire is inserted in the tibia from postero-lateral to antero-medial taking care to avoid the tibial tunnel used to reconstruct the ACL. A reamer is inserted, to breach only lateral the tibial cortex. This step can be checked using the image intensifier. Following successful breach, the tunnel is sequentially enlarged, if necessary, using the same careful technique, with larger diameter drills up to obtain a tunnel of the diameter corresponding to that of the graft. A grasper is placed underneath the ITB from the tibial incision to the femoral emergence of the ACL tunnel, and is used to shuttle the graft under the ITB (Figure 3).

A suture loop is employed by passing a $\mathrm{n}^{\circ} 2$ Ethibond suture (Ethicon) in a retrograde fashion from the antero-medial part of the tibial tunnel for the ALL to the postero-lateral portion.
The suture tails from the whipstitched end of the gracilis tendon are passed into this loop to allow the passage of the ALL graft through the tunnel.

The graft is tensioned with the knee in full extension and neutral rotation, and fixed at tibia, with a non-metallic interference screw 7 x 25 mm (RCI, Smith \& Nephew Endoscopy, Andover, Massachusetts). The excess part of the graft is eliminated. Combined ACL and ALL reconstruction is shown in figure 4.

\section{POST-OPERATIVE MANAGEMENT}

In the early postoperative period, patients should be encouraged to keep the leg elevated and to move every joint of the lower limb when not weight-bearing. Cryotherapy is encouraged.

The rehabilitation protocol is the same used in ACL reconstructions. Isometric quadriceps contraction with a straight 


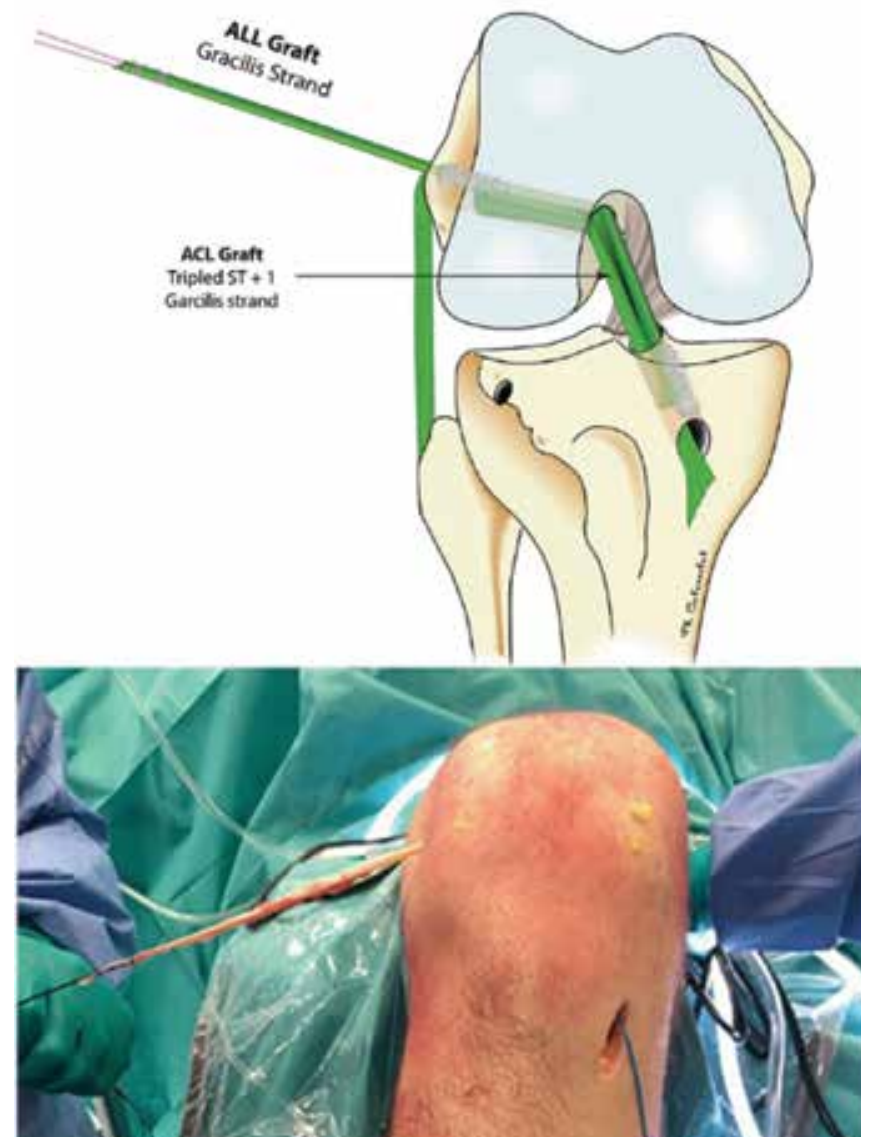

Figure 2. The part of the graft constituted by the tripled semitendinosus and the distal portion of the gracilis reconstruct the $A C L$, while the remaining proximal portion of the gracilis tendon is used to reconstruct the ALL. leg and rise with the knee extended are performed as tolerated. Patients are allowed to walk with the aid of crutches immediately after surgery. We do not use post-operative splinting of the operated knee.

\section{RISK}

Complications are similar to those encountered when the ACL reconstruction performed alone.

The reoperation rate of the combined reconstruction is comparable to the rate of reconstruction of isolated ACL and has a very low rate of complications (16). It is important not to over tension ALL during reconstruction to avoid over-constraint of the knee (17).

\section{DISCUSSION}

The primary complaint of patients with ACL insufficiency after an injury or failed reconstruction is rotational instability with pivoting or cutting activities (18).

Extra-articular reconstruction provides additional reduction of the pivot shift as compared with intra-articular reconstruction alone. This procedure allows the combined anatomical reconstruction of the ACL and the ALL, reproducing the anatomical attachment and insertion sites of the ALL (15).

The combined reconstruction of the ACL and ALL aims to eliminate knee instability to restore normal kinematics and reduce the risk of meniscal and cartilaginous damage.

The ALL pattern changes during knee flexion and tibial intra-rotation movements. Indeed, the ligament is stretched
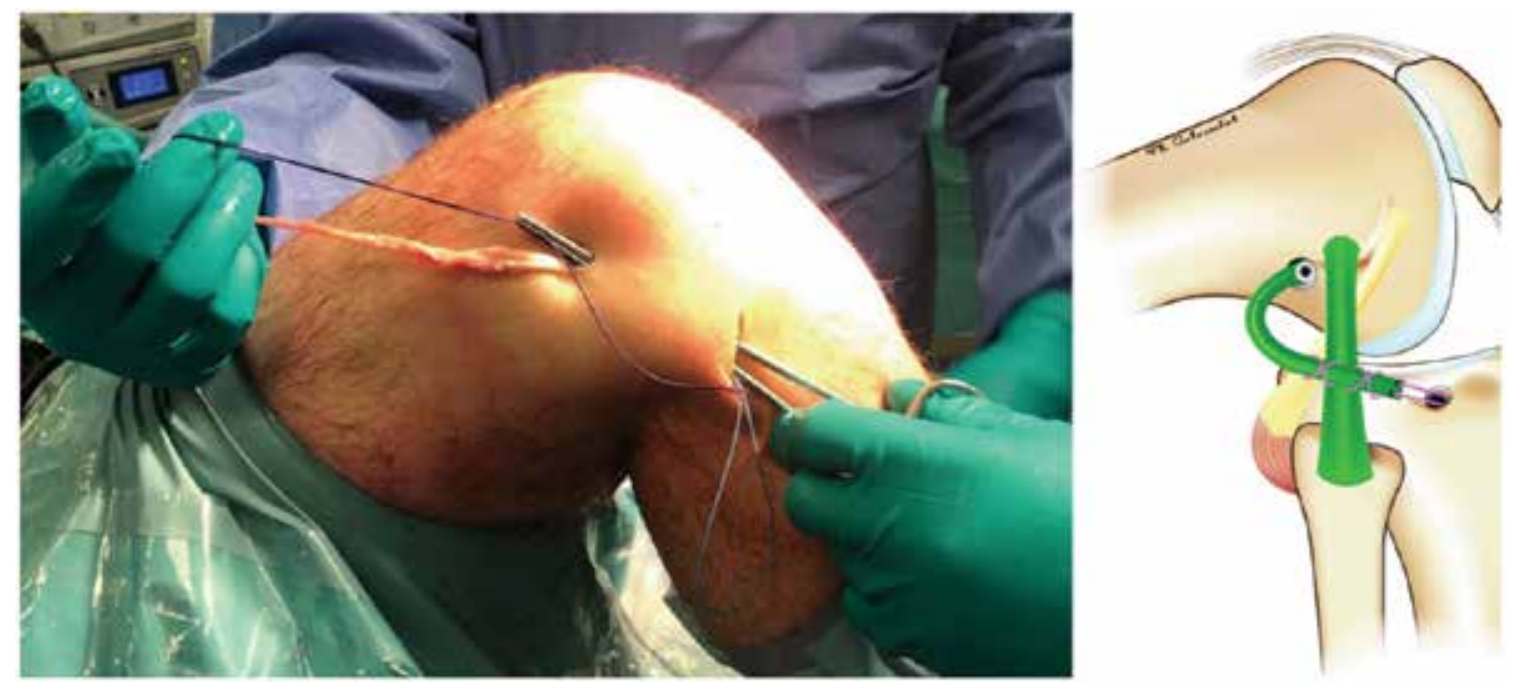

Figure 3. A grasper is placed underneath the ITB from the tibial incision to the femoral emergence of the ACL tunnel, and is used to shuttle the graft under the ITB. 


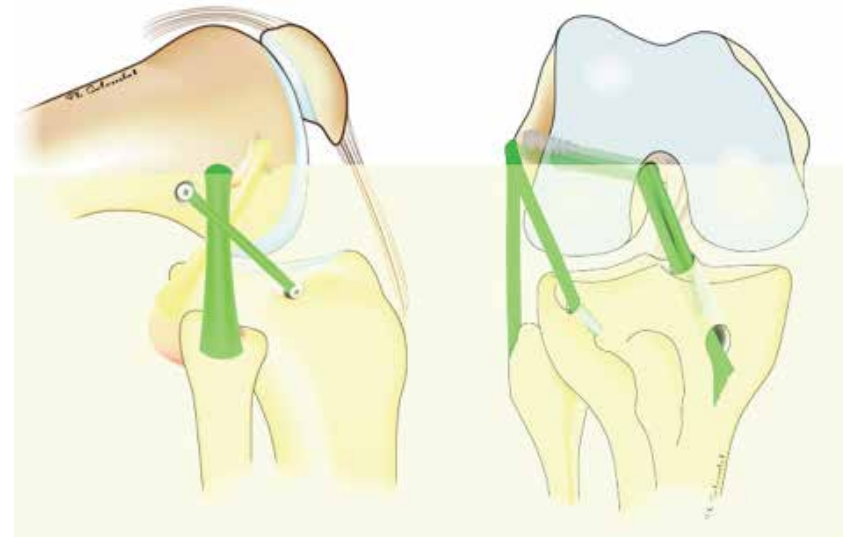

Figure 4. Final appearance of combined ACL and ALL reconstruction.

in extension and at $20^{\circ}$ in internal rotation, and relaxed with knee flexed at $120^{\circ}$ and $90^{\circ}$ in internal rotation. The femoral insertion proximal and posterior to the lateral epicondyle allows these characteristics to be reproduced (19).

The ALL inserts into the posterior $4 \mathrm{~mm}$ femur and $8 \mathrm{~mm}$ proximal to the lateral epicondyle so that it is stretched when the knee extends and allows better control of the anterolateral rotational instability (ALRI) (20).

Regarding the choice of the graft, the gracilis tendon is appropriate. Even though other artificial or autologous grafts can provide a sufficient load to replace ALL, the ultimate tension of the gracilis tendon matches the ALL (21). The maximum load to failure for the ALL was 141 N, 200.7 $\mathrm{N}$ for the duplicated gracilis, and $161.1 \mathrm{~N}$ for the ITB; the stiffness was $21 \mathrm{~N} \mathrm{~mm}^{-1}$ for the ALL, $131.7 \mathrm{~N} \mathrm{~mm}^{-1}$ for the gracilis and $39.9 \mathrm{~N} \mathrm{~mm}^{-1}$ for the ITB; the elongation at failure was $6.2 \mathrm{~mm}$ for the ALL, $19.9 \mathrm{~mm}$ for the gracilis and $20.8 \mathrm{~mm}$ for the ITB. Therefore, the tendon of gracilis had the highest maximum load to failure, while the mechanical properties of the ITB most closely resemble those of the ALL (22).

Over time, different types of lateral tenodesis have been associated with reconstruction of the ACL to counteract possible residual rotational instability of the knee. These techniques have produced mixed results, not necessary demonstrating a clear clinical improvement compared to isolated reconstruction of the ACL (23).

On the other hand, other clinical studies demonstrated better joint stability when extra-articular augmentation was associated with ACL reconstruction, such as the MacIntosh modified Coker procedure with autologous iliotibial tract (2); the over-the-top technique with a combined autologous semitendinosus and gracilis graft (24), and various techniques in combination with revision ACL reconstruc- tion (25). Recently, combined reconstruction of ACL and ALL using the gracilis tendon, have been shown to allow excellent control of rotational instability of the knee without specific complications at a minimum follow-up of 2 years (1).

Same doubts remain regarding the advantages of reconstructing the ALL. Nevertheless, a combined reconstruction should be considered in patients with a high degree of rotational ability and in ACL revision surgery. We consider the use of this procedure in elite athletes or in patients with hypermobility.

The procedure described uses the ipsilateral gracilis tendon. In this way the iliotibial band, another important structure in controlling rotational stability, is not weakened.

\section{CONCLUSION}

Combined ACL and ALL reconstruction restores rotational stability and reduces the risk of meniscal injury and the development of future knee osteoarthritis. It is also associated with no increased risk of reoperation and a very low rate of complications, contributing to a reduction in ACL graft rupture rates.

\section{REFERENCES}

1. Chambat P, Guier C, Sonnery-Cottet B, Fayard J-M, Thaunat M. The evolution of ACL reconstruction over the last fifty years. Int Orthop. 2013 Feb;37(2):181-6.

2. Vadalà AP, Iorio R, De AC, et al. An extra-articular procedure improves the clinical outcome in anterior cruciate ligament reconstruction with hamstrings in female athletes. Int Orthop Int Orthop. 2013 Feb;37, 37(2, 2):187, 187-92.

3. Hughston, JACK C., et al. "Classification of knee ligament instabilities. Part I. The medial compartment and cruciate ligaments.” JBJS 58.2 (1976): 159-172.

4. Lemaire M, Combelles F. Current technique of plastic repair with fascia lata for old tears of the anterior cruciate ligament. Rev Chir Orthop Reparatrice Appar Mot. 1980;66(8):523-5.

5. Acquitter Y, Hulet C, Locker B, Delbarre J-C, Jambou S, Vielpeau C. [Patellar tendon-bone autograft reconstruction of the anterior cruciate ligament for advanced-stage chronic anterior laxity: is an extra-articular plasty necessary? A prospective randomized study of 100 patients with five year follow-up]. Rev Chir Orthop Reparatrice Appar Mot. 2003 Sep;89(5):413-22.

6. Ferretti A. Extra-articular reconstruction in the anterior cruciate ligament deficient knee: a commentary. Joints. 2014 May $8 ; 2(1): 41-7$.

7. Sonnery-Cottet B, Saithna A, Cavalier M, et al. Anterolateral Ligament Reconstruction Is Associated With Significantly Reduced ACL Graft Rupture Rates at a Minimum Follow-up of 2 Years: A Prospective Comparative Study of 502 Patients From the SANTI Study Group. Am J Sports Med. 2017 Jun;45(7):1547-57. 
8. Sonnery-Cottet B, Thaunat M, Freychet B, Pupim BHB, Murphy CG, Claes S. Outcome of a Combined Anterior Cruciate Ligament and Anterolateral Ligament Reconstruction Technique With a Minimum 2-Year Follow-up. Am J Sports Med. 2015 Jul;43(7):1598-605.

9. Vieira ELC, Vieira EA, da Silva RT, et al. An anatomic study of the iliotibial tract. Arthrosc J Arthrosc Relat Surg Off Publ Arthrosc Assoc N Am Int Arthrosc Assoc. 2007 Mar;23(3):26974.

10. Claes S, Vereecke E, Maes M, Victor J, Verdonk P, Bellemans $\mathrm{J}$. Anatomy of the anterolateral ligament of the knee. J Anat. 2013 Oct;223(4):321-8.

11. Monaco E, Ferretti A, Labianca L, Navigated knee kinematics after cutting of the ACL and its secondary restraint. Knee Surg Sports Traumatol Arthrosc Off J ESSKA. 2012 May;20(5):870-7.

12. Parsons EM, Gee AO, Spiekerman C, Cavanagh PR. The biomechanical function of the anterolateral ligament of the knee. Am J Sports Med. 2015 Mar;43(3):669-74.

13. Padulo J, Oliva F, Frizziero A, Maffulli N. Muscles, Ligaments and Tendons Journal - Basic principles and recommendations in clinical and field Science Research: 2016 Update. Muscles Ligaments Tendons J. 2016 May 19;6(1):1-5.

14. Kennedy MI, Claes S, Fuso FAF, et al. The Anterolateral Ligament: An Anatomic, Radiographic, and Biomechanical Analysis. Am J Sports Med. 2015 Jul;43(7):1606-15.

15. Corry, I. S., Webb, J. M., Clingeleffer, A. J., \& Pinczewski, L. A. (1999). Arthroscopic reconstruction of the anterior cruciate ligament. The American Journal of Sports Medicine, 27(4), 444-454

16. Thaunat M, Clowez G, Saithna A, et al. Reoperation Rates After Combined Anterior Cruciate Ligament and Anterolateral Ligament Reconstruction: A Series of 548 Patients From the SANTI Study Group With a Minimum Follow-up of 2 Years. Am J Sports Med. 2017 Sep;45(11):2569-77.
17. Schon J, Brady A, Moatshe G, et al. Anatomic Anterolateral Ligament Reconstruction of the Knee Leads to Overconstraint at any Fixation Angle. Orthop J Sports Med [Internet]. 2016 Jul 29 [cited 2018 Mar 19];4(7 suppl4). Available from: https://www.ncbi.nlm.nih.gov/pmc/articles/PMC4968332/

18. Tashman S, Collon D, Anderson K, Kolowich P, Anderst W. Abnormal rotational knee motion during running after anterior cruciate ligament reconstruction. Am J Sports Med. 2004 Jun;32(4):975-83.

19. Imbert P, Lutz C, Daggett $M$, et al. Isometric Characteristics of the Anterolateral Ligament of the Knee: A Cadaveric Navigation Study. Arthrosc J Arthrosc Relat Surg Off Publ Arthrosc Assoc N Am Int Arthrosc Assoc. 2016 Oct;32(10):2017-24.

20. Katakura M, Koga H, Nakamura K, Sekiya I, Muneta T. Effects of different femoral tunnel positions on tension changes in anterolateral ligament reconstruction. Knee Surg Sports Traumatol Arthrosc Off J ESSKA. 2017 Apr;25(4):1272-8.

21. ZENS, Martin, et al. Mechanical tensile properties of the anterolateral ligament. Journal of experimental orthopaedics, 2015, 2.1: 7

22. Wytrykowski K, Swider P, Reina N, et al. Cadaveric Study Comparing the Biomechanical Properties of Grafts Used for Knee Anterolateral Ligament Reconstruction. Arthrosc J Arthrosc Relat Surg Off Publ Arthrosc Assoc N Am Int Arthrosc Assoc. 2016 Nov;32(11):2288-94.

23. Saithna A, Thaunat M, Delaloye JR, Ouanezar H, Fayard JM, Sonnery-Cottet B. Combined ACL and Anterolateral Ligament Reconstruction: JBJS Essent Surg Tech. 2018 Jan;8(1):e2.

24. Zaffagnini S, Marcacci M, Lo Presti M, Giordano G, Iacono F, Neri MP. Prospective and randomized evaluation of ACL reconstruction with three techniques: a clinical and radiographic evaluation at 5 years follow-up. Knee Surg Sports Traumatol Arthrosc Off J ESSKA. 2006 Nov;14(11):1060-9.

25. Trojani C, Beaufils P, Burdin G, et al. Revision ACL reconstruction: influence of a lateral tenodesis. Knee Surg Sports Traumatol Arthrosc Off J ESSKA. 2012 Aug;20(8):1565-70. 